\title{
Ultrasound guided erector spinae plane catheter for thoracotomy: a case report
}

\author{
Ali Usman, Ahsun Waqar Khan, Muhammad Khurram ljaz \\ Author affiliation: \\ Department of Anesthesiology \& Pain Management, Shaukat Khanum Memorial Cancer Hospital and Research Centre, Johar \\ Town, Lahore, Pakistan.
}

Correspondence: Ali Usman; E-mail: draliusman8166@gmail.com; Phone: +923320681667; Mobile: +92 3320681667

\section{Abstract}

Major thoracic surgery results in severe postoperative pain. Epidural analgesia is considered the gold standard for pain management after thoracic surgery. However, epidural blocks are contraindicated in certain clinical conditions. Pain management in such patients poses a unique challenge to the anesthesiologists. Erector spinae plane block (ESPB) is a less invasive and relatively new technique, which has shown promising results. This case report describes the use of ESPB as an alternate analgesic technique. A 65-year-old man underwent thoracotomy and left upper lobe resection. Intraoperative and postoperative pain was managed with intermittent boluses of bupivacaine through the erector spinae plane catheter. Effective analgesia was achieved with minimal morphine consumption in postoperative period. Although central neuraxial blocks are considered to be gold standard for major thoracic surgery but where applicable, alternatives can be opted for better patient outcomes.

Key words: Analgesia; Erector spinae plane block; Thoracotomy; Pain control; Neuraxial analgesia

Citation: Usman A, Khan AW, ljaz MK. Ultrasound guided erector spinae plane catheter for thoracotomy: a case report. Anaesth. pain intensive care 2021;25(6):812-815; DOI: 10.35975/apic.v25i6.1707

Received: August 16, 2021, Reviewed: October 28, 2021, Accepted: October 31, 2021

\section{Introduction}

Pain, the fifth vital sign, becomes even more important in the postoperative phase and is one of the most critical indicators of postoperative recovery. The prevalence of postoperative pain may reach up to $95 \%$ in the first three days, ${ }^{1}$ and optimal analgesia has shown to improve patient satisfaction and allow earlier hospital discharge. Thoracotomy is associated with severe postoperative pain. Suboptimal pain control may result in atelectasis, inadequate sputum clearance and several other respiratory complications and it may also lead to chronic post thoracotomy pain syndrome. ${ }^{2}$ Different modalities and methods used for analgesia after thoracic surgery include central and peripheral nerve blocks. Erector spinae plane block (ESPB) is a novel analgesia technique important in managing acute intraoperative and postoperative pain in thoracic and breast surgeries. ${ }^{3}$ Placing a catheter in the erector spinae plane provides prolonged pain control during the postoperative period. Intraoperative and postoperative analgesia can be administered as intermittent boluses of local anesthetic $(15-20 \mathrm{~mL})$ or continuous infusion through the catheter. ${ }^{4}$ Epidural analgesia has been described as the gold standard for controlling pain in thoracic surgery, but may not be applicable in every patient. ${ }^{5}$ In our hospital, pain control during and after thoracic surgery is routinely achieved using an epidurals. However, in this particular patient, it was contraindicated as he was on dual antiplatelet therapy. Therefore, an alternative analgesic technique was adopted. The goal was to provide adequate pain relief intraoperatively as well as in postoperative period and to minimize the need of opioids.

\section{Case Report}

A sixty-five-year-old man diagnosed to be suffering from non-squamous cell carcinoma of the lung was scheduled for left upper lobe lobectomy. Preoperative anesthesia assessment revealed a history of myocardial infarction and, a drug-eluting stent placed in his right coronary artery two years ago. He was subsequently put on dual 
antiplatelet therapy (APT), which included aspirin 75 $\mathrm{mg}$ and clopidogrel $75 \mathrm{mg}$, to prevent stent thrombosis. His functional capacity was assessed to be greater than four metabolic equivalents. His 2D echocardiogram showed no wall motion abnormality, good ejection fraction and grade 1 diastolic dysfunction. Pulmonary function tests revealed an $\mathrm{FEV}_{1} / \mathrm{FVC}$ ratio greater than 0.8 and forced vital capacity of $2.9 \mathrm{~L}$.

Epidural analgesia was contraindicated in this case, as antiplatelet therapy had only been stopped two days prior to surgery. We decided to opt an erector spinae plane block (ESPB) for pain management.

Informed written consent for general anesthesia with invasive monitoring, and ESPB with catheter insertion was obtained. After checking airway equipment, anesthesia machine and preparing anesthesia and emergency medicines, standard monitors was applied to the patient. General anesthesia was induced with fentanyl $100 \mu \mathrm{g}$, incremental doses of propofol until the patient was asleep and atracurium $0.5 \mathrm{mg} / \mathrm{kg}$. An arterial line was placed in the left radial artery for invasive blood pressure monitoring. The airway was secured with a $37 \mathrm{~F}$ double-lumen left-sided tube using a McGrath blade No. 4. Subsequently, the patient was placed in the right lateral position, and the ESPB site was sterilized using chlorohexidine. Thoracic vertebrae level seven (T7) was identified using the landmark technique, and the spinous process of T6 was identified using ultrasound. The transverse process of the vertebra was identified anout $3 \mathrm{~cm}$ from the midline. A linear ultrasound probe with a frequency of $10 \mathrm{MHz}$ was used. An $18 \mathrm{G}$ Tuohy needle (PORTEX® epidural set) was inserted under ultrasound guidance, using the in-plane technique, and the transverse process was hit. The needle was withdrawn $1 \mathrm{~mm}$, after hydro-dissecting the erector spinae plane with $5 \mathrm{ml}$ of $2 \%$ lidocaine (Figure 1), 20 $\mathrm{mL}$ of $0.25 \%$ bupivacaine was administered under ultrasound visualization. An epidural catheter was inserted in this plane through the Tuohy needle (Figure 2). The catheter at skin was secured at $11 \mathrm{~cm}$ mark with a LOCKIT Plus® catheter securement device (Smiths Medical) after removing the needle.

Patient underwent left upper lobe excision uneventfully. The duration of surgery was $118 \mathrm{~min}$ with estimated blood loss of approximately $500 \mathrm{ml}$. The patient remained hemodynamically stable throughout the procedure. He was administered 1.5 L of Ringer lactate crystalloid solution intravenously. At the end of the procedure, inj. paracetamol $1 \mathrm{gm}$ was administered as a co-analgesic. Patient was extubated in the operating room and shifted to the post-anesthesia care unit (PACU).

In the PACU, the patient became fully awake and

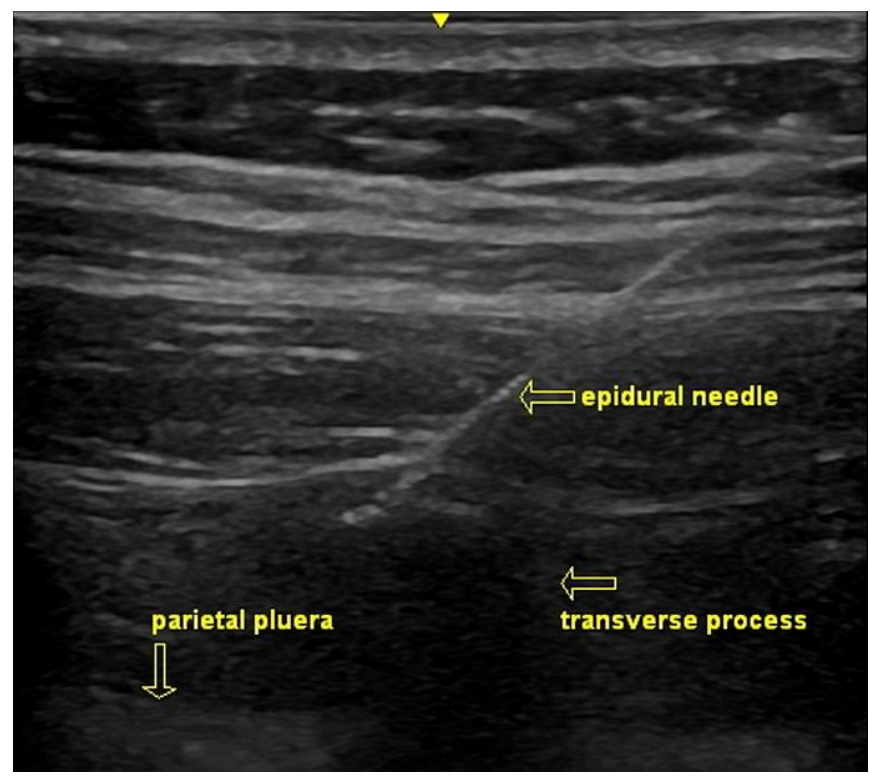

Figure 2: Epidural needle hitting the transverse process and erector spinae plane

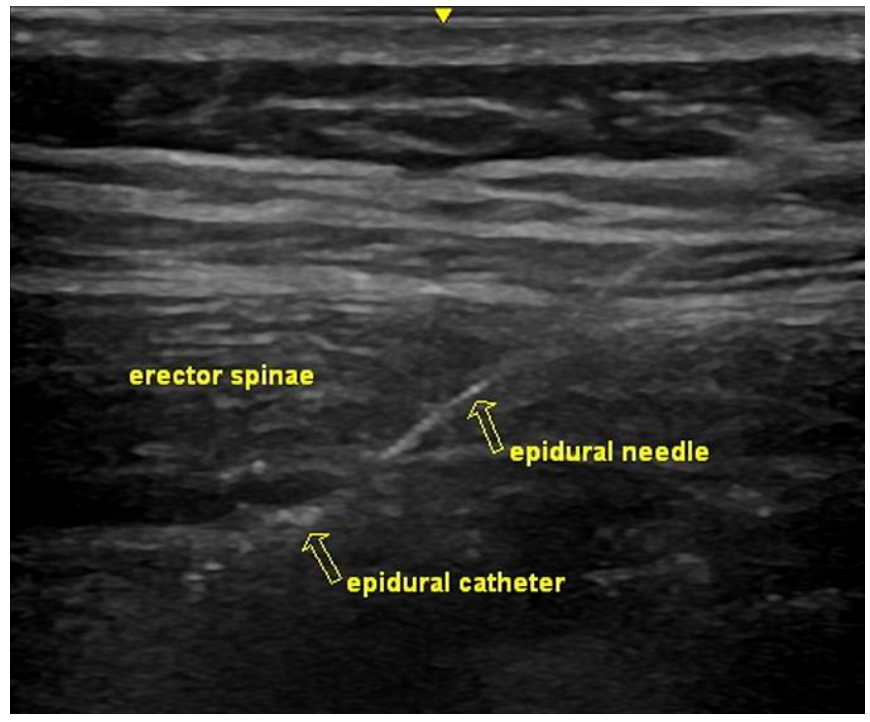

Figure 1:Epidural catheter after hydro-dissection of the erector spinae plane block

received an additional $20 \mathrm{~mL}$ of bupivacaine $0.25 \%$ through the catheter. He remained pain free during his two hours stay in PACU. His numerical rating score (NRS) was $2 / 10$ at the time of discharge to the high dependency unit. During the next $24 \mathrm{~h}$, patient's pain was managed by six hourly boluses of $20 \mathrm{ml}$ of bupivacaine $0.125 \%$. During the following days, the patient's pain was controlled with boluses of bupivacaine $0.125 \%$, supplemented with inj. paracetamol. In case of breakthrough pain, morphine $2 \mathrm{mg}$ intravenous was used as a rescue analgesic. Total rescue analgesia consumption was $2 \mathrm{mg}$ of morphine during the first $24 \mathrm{~h}$. The catheter was removed on postoperative day 3 , and the patient 
resumed his antiplatelet medications afterwards. No local or systemic complications related to the ESPB were observed and the rest of the postoperative course was uneventful. He was discharged on the sixth postoperative day.

\section{Discussion}

Thoracic surgeries are associated with severe postoperative pain. Inadequate pain control in the postoperative period causes multiple problems including poor physical function, associated co-morbidities, prolonged opioid use, delayed recovery and the development of chronic postsurgical pain. Different modalities, including epidural, paravertebral, and serratus anterior plane blocks have been used to control pain. Epidural route remains the gold standard for pain control in major thoracic surgeries. A catheter is inserted in the epidural space and local anesthetics and opioids are administered through it, which act mainly by blocking the spinal nerve roots and paravertebral nerves. However, performing an epidural block in a patient taking antiplatelet medication poses the risk of epidural hematoma formation. ${ }^{6}$ Systemic opioids are also good analgesics but the associated excessive sedation and respiratory depression especially after thoracic surgery can be deleterious. These risks can be prevented by performing an ESPB, which is a safe and effective alternative pain control technique. Effective analgesia is even more important in patients having ischemic heart disease like our patient. Poorly controlled pain can result in sympathetic stimulation causing tachycardia and hypertension, which can have detrimental effects on myocardial oxygen demand and supply balance.

The erector spinae is a group of muscles on the dorsal side of the trunk that originates caudally and runs cranially. It originates from the spinous processes of the T9-T12 thoracic vertebrae and medial edge of the dorsal segment of the iliac crest. It is inserted at the spinous processes of the $\mathrm{T} 1$ and $\mathrm{T} 2$ thoracic vertebrae and the cervical vertebrae. The ESPB is an easy and efficacious regional block technique used in patients undergoing thoracic, abdominal, and breast surgeries. The mechanism of the block is not entirely understood; however, the deposition of local anesthetic anterior (deep) to the muscle reaches the paravertebral space through the ligaments and soft tissue, where it blocks the ventral, dorsal, and communicant rami of the spinal nerves, as shown in a cadaver model by Chin et al. ${ }^{7}$ and Adhikary et al. ${ }^{8}$ Other proposed mechanisms included epidural spread, as some studies have shown the contralateral spread of the local anesthetic. ${ }^{9-12}$

Performing the block under ultrasound imaging needs familiarity with the equipment and the anatomy of the structures. Ultrasound allows visualization of the anatomic planes as well as the visualization of the block needle in motion. This has remarkably increased the safety and the efficacy of the regional blocks. It also results in less needle pricks, lower dose requirements and lesser complications. ${ }^{13}$ Ultrasound guided hydrodissection is very important, as it opens up the plane and aids in catheter insertion as shown in the diagram. This can be achieved by administering a large volume (20-25 $\mathrm{mL}$ ) of local anesthetic in the erector spinae plane. The tip of the multi-orifice catheter is placed under direct visualization at the desired level. Other drugs such as opioids, alpha-2 agonists, steroids, and non-steroidal anti-inflammatory drugs can also be used along with the local anesthetic to increase both the density and duration of the block. One meta-analysis including 11 randomized control trials and 679 patients, showed no major complications with the erector spinae block. ${ }^{14}$

The ESPB is a reasonable alternative analgesic modality, whenever the central neuraxial blocks are contraindicated. It offers the advantage of decreased incidence of the side effects and the complications as compared to the central neuraxial blocks. Our experience with ESPB was encouraging. The patient did not experience any side effects or complications. He remained pain free during his postoperative course.

\section{Conclusion}

Ultrasound guided erector spinae plane block is a safe and effective technique for post thoracotomy pain management. It is ideal for patients when central neuraxial blocks are contraindicated. Catheter placement in the erector spinae plane allows the administration of boluses or continuous infusion of local anesthetics, maintaining adequate analgesia for several days after surgery.

\section{Conflict of interests}

The authors had no conflict of interests in this case report. No funding was involved.

\section{Authors" contribution}

All authors took equal part in the conduction of the study work and manuscript editing

\section{References}

1. Singh PK, Saikia P, Lahakar M. Prevalence of acute postoperative pain in patients in adult age-group undergoing inpatient abdominal surgery and correlation of intensity of pain and satisfaction with analgesic management: A crosssectional single institute-based study. Indian J Anaesth. 2016;60(10):737-743. [PubMed] DOI: 10.4103/00195049.191686 
2. A Mesbah, J Yeung, F Gao. Pain after thoracotomy. BJA Educ. 2016;16(1):1-7. DOI: 10.1093/bjaceaccp/mkv005

3. Jain K, Jaiswal V, Puri A. Erector spinae plane block: Relatively new block on horizon with a wide spectrum of application - A case series. Indian J Anaesth. 2018;62:80913. [PubMed] DOI: 10.4103/ija.IJA_263_18

4. Ince I, Ozmen O, Aksoy M, Zeren S, Ulas AB, Aydin Y. Erector Spinae Plane Block Catheter Insertion under Ultrasound Guidance for Thoracic Surgery: Case Series of Three Patients. Eurasian J Med. 2018;50:204-6. [PubMed] DOI: 10.5152/eurasianjmed.2018.18147

5. El-Tahan MR. Role of thoracic epidural analgesia for thoracic surgery and its perioperative effects. J Cardiothorac Vasc Anesth. 2017;31:1417-26. [PubMed] DOI: 10.1053/j.jvca.2016.09.010

6. Cook TM, Counsell D, Wildsmith JA, et al. Major complications of central neuraxial block: report on the Third National Audit Project of the Royal College of Anaesthetists. Br J Anaesth. 2009;102:179-90. [PubMed] DOI: 10.1093/bja/aen360

7. Chin KJ, Malhas L, Perlas A. The erector spinae plane block provides visceral abdominal analgesia in bariatric surgery: a report of 3 cases. Reg Anesth Pain Med. 2017:42:372-6. 10.1097/AAP.0000000000000581

8. Adhikary SD, Bernard S, Lopez H, Chin KJ. Erector Spinae Plane Block Versus Retrolaminar Block: A Magnetic Resonance Imaging and Anatomical Study. Reg Anesth Pain Med. 2018 Oct;43(7):756-762. [PubMed] DOI: 10.1097/AAP.0000000000000798

9. Ivanusic J, Konishi Y, Barrington MJ. A cadaveric study investigating the mechanism of action of erector spinae blockade. Reg Anesth Pain Med. 2018 Aug;43(6):567-571. [PubMed] DOI: 10.1097/AAP.0000000000000789
10. Altıparmak B, Korkmaz Toker M, Uysal Al, Kuşçu Y, Gümüş Demirbilek S. Ultrasound-guided erector spinae plane block versus oblique subcostal transversus abdominis plane block for postoperative analgesia of adult patients undergoing laparoscopic cholecystectomy: Randomized, controlled trial. J Clin Anesth. 2019 Nov;57:31-36. [PubMed] DOI: 10.1016/j.jclinane.2019.03.012

11. Schwartzmann A, Peng $P$, Maciel MA, Forero $M$. Mechanism of the erector spinae plane block: insights from a magnetic resonance imaging study. Can J Anaesth. 2018;65:1165-6. [PubMed] DOI: 10.1007/s12630-018$1187-y$

12. Tulgar S, Selvi O, Ahiskalioglu A, Ozer Z. Can unilateral erector spinae plane block result in bilateral sensory blockade?. Can J Anaesth. 2019;66:1001-2. [PubMed] DOI: 10.1007/s12630-019-01402-y

13. Koscielniak-Nielsen ZJ. Ultrasound-guided peripheral nerve blocks: what are the benefits? Acta Anaesthesiol Scand. 2008 Jul;52(6):727-37. [PubMed] DOI: 10.1111/j.1399-6576.2008.01666.x

14. Zhang Y, Liu T, Zhou Y, Yu Y, Chen G. Analgesic efficacy and safety of erector spinae plane block in breast cancer surgery: a systematic review and meta-analysis. BMC Anesthesiol. 2021;21:59. [PubMed] DOI: 10.1186/s12871021-01277-x

15. De la Cuadra-Fontaine JC, Concha M, Vuletin F, Arancibia H. Continuous Erector Spinae Plane block for thoracic surgery in a pediatric patient. Paediatr Anaesth. 2018;28:74-5. [PubMed] DOI: 10.1111/pan.13277

16. Khali AE, Abdallah NM, Bashandy GM, Kaddah TA. Ultrasound-Guided Serratus Anterior Plane Block Versus Thoracic Epidural Analgesia for Thoracotomy Pain. J Cardiothorac Vasc Anesth. 2017;31:152-8. [PubMed] DOI: 10.1053/j.jvca.2016.08.023 